\title{
Dificuldades na Avaliaçáo Neuropsicológica de Pacientes com Esclerose Múltipla
}

\author{
Difficulty In The Neuropsychological Evaluation of Patients With Multiple Sclerosis
}

\author{
Sabrina Martins Barroso ${ }^{1}$, Sonia Beatriz Felix Ribeiro ${ }^{2}$, Maria Cristina \\ Itagiba Fonseca ${ }^{3}$, Débora Prado da Silva ${ }^{4}$
}

\section{RESUMO}

Objetivo. A avaliação neuropsicológica pode auxiliar no diagnóstico e tratamento de pacientes com transtornos neurodegenerativos, mas ainda enfrenta problemas com a escolha e a qualidade dos instrumentos, especialmente ao avaliar patologias específicas, como a Esclerose Múltipla (EM). O objetivo do presente trabalho é apresentar os resultados e as dificuldades enfrentadas ao realizar a avaliação neuropsicológica de pacientes com EM atendidos em um ambulatório público de uma cidade do interior de Minas Gerais. Método. Aplicação de bateria de avaliação neuropsicológica em 24 pacientes com EM. Resultados. A maioria dos pacientes apresentou algum déficit nas funçôes executivas, especialmente na memória ou na capacidade motora. O processo de avaliação evidenciou a dificuldade para utilizar provas de longa duração em pacientes que sofrem de fadiga crônica e para obter material validado específico para avaliar pacientes com EM. Conclusóes. É necessário avaliar os pacientes no período da manhã, com instrumentos menos extensos e também avaliar o estado emocional dos pacientes com EM.

Unitermos. Esclerose Múltipla, Neuropsicologia, Avaliação da Deficiência, Sinais e Sintomas, Testes Psicológicos.

Citaçáo. Barroso SM, Ribeiro SBF, Fonseca MCI, Silva DP. Dificuldades na Avaliação Neuropsicológica de Pacientes com Esclerose Múltipla.

\begin{abstract}
Objective. Neuropsychological assessment can assist in the diagnosis and treatment of patients with neurodegenerative disorders, but still faces problems with the choice and quality of the instruments, especially when evaluating specific diseases such as Multiple Sclerosis (MS). The aim of this paper is to present the results and the difficulties faced when conducting neuropsychological assessment of MS patients from an outpatient clinic of a public city in Minas Gerais. Method. Application of neuropsychological assessment battery in 24 patients with MS. Results. Most patients showed some deficits in executive functions, especially in memory or motor skills. The evaluation showed evidence a difficulty to long term use in patients suffering from chronic fatigue and for specific equipment validated to assess patients. Conclusions. It is necessary to evaluate patients in the morning, with less extensive tools and also assess the emotional state of patients with MS.
\end{abstract}

Keywords. Multiple Sclerosis, Neuropsychology, Disability Evaluation, Signs and Symptoms, Psychological Tests.

Citation. Barroso SM, Ribeiro SBF, Fonseca MCI, Silva DP. Difficulty In The Neuropsychological Evaluation of Patients With Multiple Sclerosis.

\footnotetext{
Trabalho realizado na Universidade Federal do Triângulo Mineiro, Uberaba-MG, Brasil.

1.Psicóloga, Doutoranda em Saúde Pública pela Universidade Federal de Minas Gerais (UFMG), Professora da Universidade Federal do Triângulo Mineiro (UFTM), integrante do Grupo de Estudo e Pesquisa em Esclerose Múltipla da UFTM (GEPEM-UFTM), Uberaba, MG, Brasil.

2.Médica, Doutora em Medicina pela Universidade de São Paulo. Líder do Grupo de Estudo e Pesquisa em Esclerose Múltipla da UFTM (GEPEM-UFTM), Uberaba, MG, Brasil.

3.Estudante de Psicologia (UFTM). Estagiária de Iniciação Científica, integrante do GEPEM-UFTM, Uberaba, MG, Brasil.

4.Estudante de Psicologia (UFTM), Estagiária de Iniciaçáo Científica, integrante do Laboratório de Avaliação das Diferenças Individuais (LADI-UFTM), Uberaba, MG, Brasil.
}

Endereço para correspondência: Sabrina M. Barroso Universidade Federal do Triângulo Mineiro Av. Getúlio Guaritá, 159, sala 320 - Bairro Abadia CEP 38025-440, Uberaba-MG, Brasil. E-mail: sabrina@psicologia.uftm.edu.br 


\section{INTRODUÇÃO}

A Esclerose Múltipla (EM) é uma doença progressiva, autoimune e de etiologia desconhecida. Esta doença se caracteriza por uma disfunção do sistema nervoso que causa a perda da bainha de mielina que envolve os neurônios, associada a uma inflamação e à destruição dos axônios, prejudicando a condução dos impulsos nervosos e gerando sintomas físicos e cognitivos ${ }^{1}$.

A EM não afeta igualmente homens e mulheres, sendo sua prevalência cerca de três vezes maior entre as mulheres ${ }^{1}$. No Brasil, sua prevalência foi estimada em 15/100.000 habitantes, ficando em 20.1/100.000 para o sexo feminino e 8.5/100.000 para o sexo masculino. Estes dados colocam o Brasil como um país de prevalência média para a $\mathrm{EM}^{2}$. O início da patologia antes dos 15 anos ou após os 50 anos é raro, sendo a faixa etária mais comum para o surgimento da esclerose múltipla a dos jovens adultos (20 a 40 anos) ${ }^{3}$.

A EM possui quatro manifestaçóes clínicas distintas $^{4}: 1$. Forma recorrente remitente: em que os sintomas costumam exacerbar-se por cerca de uma ou duas semanas (período de crise), seguidos por uma melhora gradual, com duração de dois ou três meses. No início da doença, a recuperação dos sintomas pode ser total durante o período de remissão, contudo, com a repetição das crises, alguns déficits passam a se acumular; 2. Forma Progressiva Secundária: nesse quadro a doença inicia como forma surto-remissiva, mas com o decorrer do tempo os comprometimentos tornam-se progressivos, sem fases de melhora dos sintomas; 3. Forma Progressiva Primária: em que o comprometimento progressivo está presente desde o início da doença; 4. Forma Progressiva com Surtos: quando a doença se mostra progressiva desde o surgimento, apresenta surtos claros de exacerbação dos sintomas, com ou sem recuperação total das funções afetadas e piora progressiva fora dos momentos de surto.

Independente da forma de manifestação, os sintomas da EM incluem comprometimentos motores (paralisias, tremores, parestesias, dores, etc.), alteraçóes emocionais (depressão, estresse, ansiedade exacerbada) e cognitivas (déficits de memória de trabalho, atenção, funções executivas, velocidade de processamento da informação, etc.). A fadiga, não relacionada à fraqueza e a piora dos sintomas durante exercícios físicos ou exposi- ção a altas temperaturas são bastante frequentes, gerando considerável impacto psicossocial e afetando a qualidade de vida dos pacientes ${ }^{1,3,5}$.

Com relação aos déficits cognitivos, estudos indicam que entre 50 e $65 \%$ dos pacientes com EM possuem algum grau de déficit cognitivo ${ }^{4,6}$. Ao longo das últimas décadas o interesse em investigar as dificuldades cognitivas dos pacientes com EM, assim como o interesse por desenvolver intervençôes capazes de minimizar déficits nas funçóes executivas desses pacientes aumentou significativamente ${ }^{5}$. Em levantamento bibliográfico ${ }^{4}$ realizado em 2009 são elencados diversos estudos sobre os aspectos cognitivos de pacientes com EM realizados na América Latina, nos quais se observou que as avaliaçóes mais frequentes envolveram funçóes executivas, memória e o desenvolvimento de instrumentos de avaliação cognitiva.

No Brasil, ainda há poucos estudos sobre o perfil cognitivo dos pacientes com EM. Um estudo ${ }^{5}$ investigou o curso evolutivo da EM, tendo identificado um perfil heterogêneo, tanto para os déficits apresentados, quanto para o prognóstico. A variabilidade de manifestaçóes faz com que um diagnóstico e acompanhamento detalhado se façam necessários e, nesse sentido, as avaliaçóes neuropsicológicas podem contribuir para refinar o conhecimento sobre o quadro clínico dos pacientes. Outros trabalhos focam sua atenção para os déficits de memória, déficits motores e nas funçôes executivas ${ }^{7-10}$.

Por meio da avaliação neuropsicológica obtém-se uma visão acurada sobre as funçôes executivas, alteraçôes de personalidade e potenciais déficits na memória, atenção, linguagem e velocidade de processamento de informações, além de informaçóes sobre capacidades preservadas ou mesmo recuperadas por meio de planos de intervenção específicos. Essas informações podem ajudar a obter um diagnóstico clínico mais preciso e também são importantes para construir intervenções clínicas para pessoas com doenças crônicas, entre elas a $\mathrm{EM}^{4}$.

Ao avaliar pessoas com EM, seja com finalidade clínica ou de pesquisa, os profissionais da neuropsicologia têm enfrentado algumas dificuldades inerentes à avaliação psicológica no Brasil e outras diretamente relacionadas com esta patologia. Com relaçấo aos instrumentos para realizar avaliaçôes neuropsicológicas, há uma substancial carência de material validado para a realidade bra- 
sileira. Esse aspecto se agravou nos últimos anos, pois o Conselho Federal de Psicologia (CFP), órgão responsável por regular a definição do que seja um teste psicológico, sua criação, comercialização e utilização dos testes psicológicos no Brasil ${ }^{11-13}$ limitou judicialmente a utilização de instrumentos validados de avaliação neuropsicológica aos profissionais da Psicologia.

Além disso, o CFP, por meio do código de ética profissional da categoria, proíbe aos psicólogos utilizar em suas avaliaçóes instrumentos não validados para o contexto brasileiro, sob pena de puniçóes que podem envolver desde multas até a eventual cassação do direito ao exercício profissional ${ }^{14}$. Assim, embora autorizados a fazer uso dos testes neuropsicológicos validados, os psicólogos esbarram na ausência de material, visto que a maior parte do instrumental em neuropsicologia ainda não passou por análises psicométricas, sendo construídos e mantidos pela aparente relevância clínica.

Embora vivências frequentes para os neuropsicólogos clínicos, o conhecimento sobre os instrumentos de avaliação disponíveis, sobre os aspectos a considerar para compor as baterias de avaliação e as possíveis dificuldades para realizar avaliaçóes em portadores de patologias específicas - como é o caso da esclerose múltipla - são aspectos pouco abordados nos artigos sobre avaliação neuropsicológica. Esse foi um dos motivadores para o presente trabalho, cujo objetivo é apresentar os resultados de uma avaliaçâo neuropsicológica em pacientes com EM, destacando as principais dificuldades enfrentadas pelos profissionais da neuropsicologia durante essas avaliaçóes.

O recorte do presente trabalho foca na escolha metodológica e dificuldades vivenciadas para conduzir processos de avaliação neuropsicológica de pacientes com EM, por este ser um aspecto pouco abordado nas publicaçóes da área. Considerando esse recorte, optou-se por abordar apenas superficialmente os resultados da avaliação neuropsicológica em si e apresentar maior detalhamento sobre o processo avaliativo, ficando para publicaçóes posteriores o detalhamento dos déficits cognitivos observados nos pacientes.

\section{MÉTODO}

\section{Amostra}

24 pacientes com diagnóstico clínico de Esclerose
Múltipla atendidos em um ambulatório público vinculado a um hospital universitário de uma cidade de médio porte (300 mil habitantes), localizada no interior do estado de Minas Gerais. Todos os participantes possuíam mais de 18 anos e assinaram o termo de consentimento livre e esclarecido.

\section{Instrumentos}

Optou-se por utilizar uma bateria de avaliação de ampla e já em utilização entre neuropsicólogos clínicos no Brasil. Esta decisão visou garantir a qualidade do instrumental e permitir intercâmbio de conhecimento e comparação de resultados entre profissionais de diferentes instituições. Para a escolha dos testes, duas instituições foram previamente consultadas: o Hospital das Clínicas de São Paulo e a Universidade Federal de Minas Gerais. Seguindo a indicação dos testes utilizados por essas instituiçóes, a bateria adotada na presente avaliação foi composta por: Teste do Relógio, Questionário de Queixa de Memória, Testes de listas de palavras - recordaçáo imediata e recordação tardia, Testes Práticos de Função Frontal, Bateria de Avaliação da Memória de Trabalho (BAMT-UFMG) e a Multiple Sclerosis Functional Composite Measure (MSFC). Esses dois últimos instrumentos foram desenvolvidos especialmente para a avaliação da memória e das funçóes executivas de pacientes com EM e encontram-se validados para o Brasil ${ }^{15,16}$.

Cabe destacar que o teste do relógio apresenta mais de uma forma possível para correção e que na avaliação dos pacientes do presente estudo utilizou-se a correção indicada por Sunderland ${ }^{17}$, por permitir que a pontuação dos desenhos dos relógios seja feita após encerrada a aplicação de todos os testes.

\section{Análise de Dados}

Os resultados brutos dos pacientes em cada teste foram convertidos em percentis e analisados de forma descritiva, por meio de médias e porcentagem. Foram analisadas, também, dificuldades específicas que surgiram ao longo do processo avaliativo para a condução dos instrumentos. Essas análises foram conduzidas de forma qualitativa, buscando identificar suas causas e construir mecanismos compensatórios, que viabilizassem a aplicação dos testes em pacientes com EM. 
Por envolver um projeto de pesquisa e extensão, antes que qualquer contato com os pacientes fosse iniciado, o trabalho foi submetido e aprovado pelo Comitê de Ética em Pesquisa da Universidade Federal do Triângulo Mineiro (Protocolo 1633/2010).

\section{RESULTADOS}

Os resultados mostraram que a idade média dos pacientes foi de 42,46 anos $(\mathrm{DP} \pm 12,41)$ e que a maior parte dos participantes era do sexo feminino $(66,7 \%)$ e possuía ensino médio completo (33\%). A forma clínica mais prevalente foi do tipo recorrente-remitente $(79,2 \%)$, seguida da forma secundariamente progressiva $(12,5 \%)$ e primariamente progressiva $(8,3 \%)$.

Os resultados da avaliação neuropsicológica indicaram que dos 24 pacientes avaliados, seis (25\%) apresentavam déficit de memória e 15 (62,5\%) apresentavam algum prejuízo nas funções executivas de planificação, organização espacial, evocação e aprendizagem. Além desses déficits, 36,5\% (oito pacientes) apresentavam, ainda, déficit para deambular e 30,4\% apresentavam déficit no controle dos membros superiores. Esses resultados foram repassados ao hospital em que os pacientes recebem acompanhamento e passaram a embasar um projeto interventivo visando minimizar os danos cognitivos apresentados pelos pacientes, que foi executado entre os meses de março e dezembro de 2012.

Com relação à aplicação dos instrumentos, as provas clínicas utilizadas (Questionário de Queixa de Memória, Testes de listas de palavras, Testes Práticos de Função Frontal) mostraram-se rápidas e de fácil aplicação. Os pacientes entendiam as tarefas a serem realizadas e os resultados permitiram identificar a presença de problemas cognitivos mais graves, que o Mini-Exame de Estado Mental (MEEM) não havia sido capaz de identificar. De forma similar, a MFSC e o teste do relógio foram respondidos pelos pacientes com bons níveis de compreensão e sem indícios de fadiga. Ambos os testes são validados e notoriamente úteis para avaliar funçôes executivas. Embora tenham realizado a tarefa, os pacientes com EM apresentaram maiores dificuldades de compreensão e piores resultados no subteste PASAT, que compóe a MFSC. Esse resultado, contudo, já era esperado, uma vez que esse subteste envolve a resolução de contas matemáticas e a retenção de informações numéricas simultaneamente.

Durante o processo de avaliação, apenas a BAMT-UFMG representou uma dificuldade para implementar a avaliação dos pacientes com EM. Apesar de construída especialmente para essa população e conseguindo avaliar dificuldades importantes de memorização, a bateria BAMT-UFMG, devido a sua longa extensão, representou um problema para a avaliação de pacientes com EM, gerando desconforto e fadiga nos pacientes. A aplicação desse instrumento é dividida em duas sessôes de cerca de uma hora e meia cada, com realização contínua de atividades de raciocínio, o que é um tempo excessivo para pacientes que sofrem com fadiga crônica.

\section{DISCUSSÃO}

O presente estudo serviu para embasar a construção de um plano interventivo para os pacientes com EM avaliados e para explicitar dificuldades específicas para avaliar tais pacientes. Entre os instrumentos escolhidos para a bateria de avaliação, as provas clínicas indicadas pelo Hospital das Clínicas de São Paulo mostraram-se de fácil aplicação e correção. Além disso, essas provas são de rápida execução, o que favorece sua utilização em contexto ambulatorial. Mas, embora apresentem vantagens de rapidez e facilidade de aplicação, essas provas carecem de estudos sistemáticos sobre sua validade e fidedignidade. A maioria das provas clínicas, incluindo as utilizadas pela presente avaliação, é de origem norte-americana e sua utilização fora dos Estados Unidos se sustenta pela prática e pela percepçáo dos profissionais sobre a utilidade dos resultados obtidos com esses testes. Contudo, essas características não atestam a fidedignidade, poder discriminativo e nem a sensibilidade dos instrumentos aos déficits cognitivos dos pacientes. Buscamos por estudos sobre as qualidades psicométricas das provas clínicas adotadas no Brasil, contudo, não conseguimos localizar nenhuma investigação psicométrica sobre tais provas clínicas em contexto brasileiro.

Como já indicado, a utilização de material sem validação não pode ser feita por profissionais da Psicologia para avaliação profissional, exceto em contexto de pesquisa. Como a avaliação que realizamos estava vinculada a uma pesquisa, a utilização dessas provas não feriu nenhum preceito ético, contudo, a utilização desses 
instrumentos em contexto hospitalar, com finalidade unicamente clínica, pode representar um problema. Em contrapartida, sua não utilização deixa os profissionais da área sem recursos técnicos para o contexto ambulatorial.

O teste do relógio possui estudos sobre suas qualidades psicométricas ${ }^{18}$ e na experiência de avaliação aqui comentada mostrou-se sensível para avaliar pacientes com EM e identificar déficits na capacidade de compreensão, planificação, organização espacial e ordenação. Sobre esse instrumento, o principal ponto a ser considerado é a existência de mais de uma forma de correção possível, com diferentes níveis de sensibilidade às alterações cognitivas, exigindo que o profissional conheça as distintas possibilidades de correção, antes de escolher a que melhor atende a suas necessidades.

Ao aplicar a BAMT-UFMG, um dos poucos instrumentos neuropsicológicos construídos especificamente para os pacientes com EM no Brasil, enfrentamos alguns desafios. Como já indicado, este teste é longo, exige duas sessôes de uma hora e meia cada para sua aplicação e que, na indicação dos autores que validaram o instrumento, precisam acontecer dentro do prazo de sete dias corridos $^{15}$. A extensão do teste representa um problema ao avaliar uma população cujo principal sintoma é a fadiga. Além disso, em nossa experiência, vivenciamos um número alto de esquecimento dos horários agendados para avaliação por parte dos pacientes, sendo necessário reagendar e o período máximo de sete dias entre as aplicaçôes da BAMT-UFMG dificultaram os reagendamentos. Em estudos posteriores realizados pelos autores do instrumento $^{19}$, o tempo para aplicação entre a primeira e a segunda sessão de aplicação da BAMT-UFTM foi ampliado para 15 dias, aparentemente sem prejuízo da qualidade da avaliação. Essa ampliação do prazo pode representar um ganho para os avaliadores, por permitir maior flexibilidade para marcação das sessóes de avaliação e o manejo da fadiga.

$\mathrm{Na}$ avaliação aqui relatada foi necessário suspender a aplicação da BAMT-UFMG nos casos em que os pacientes mostraram-se extenuados e quando os pacientes residiam em outras cidades. Como o trabalho foi realizado em um ambulatório público, vários pacientes atendidos são de baixa renda e dependem de transporte fornecido pelas prefeituras de suas cidades para se deslocar até o hospital. Esses pacientes geralmente comparecem ao hospital cerca de uma vez por mês, apenas para avaliação médica, o que dificulta a atuação de outros profissionais de saúde, que dependem de atendimentos menos espaçados para desenvolver um trabalho interventivo. Essa frequência de comparecimento, somada à limitação de tempo da BAMT-UFMG tornaram inviável utilizar este instrumento para avaliá-los.

A BAMT-UFMG apresenta a vantagem de ser cedida gratuitamente para utilização por seus desenvolvedores, mediante solicitação. Saber que a BAMT-UFMG passou por estudos de validação e fidedignidade, bem como seu acesso gratuito, representam pontos positivos para a adoção desse instrumento nas avaliaçóes neuropsicológicas. Entretanto, erros no gabarito de correçáo fornecido pelos autores foram fonte de problemas para nossa avaliação. No material sobre o teste fornecido pelos desenvolvedores da bateria foram localizados três erros no gabarito de correçáo (BAMT - Caderno B: $5^{\text {a }}$ questão da página $19,5^{\mathrm{a}}$ questão da página 24 e $8^{\mathrm{a}}$ questão da página 30). Esses erros, se não identificados em tempo hábil, comprometem a confiabilidade das avaliaçóes, podendo induzir a conclusôes errôneas.

Os problemas para avaliar pacientes advindos de hospitais no Brasil não se restringem às escolhas de instrumentos de avaliação. Durante nossa prática, outro complicador relativo à fonte de informaçóes foi identificado. Estudos anteriores sobre a $\mathrm{EM}^{8-10}$ já haviam demonstrado que fatores como idade, escolaridade, idade de início da doença, anos de evolução e forma clínica da esclerose múltipla interferem no curso da doença e podem funcionar como variáveis preditoras do desempenho cognitivo dos pacientes $^{3}$. Para realizar a avaliação neuropsicológica, informações pessoais e de início do transtorno foram procuradas nos prontuários dos pacientes, mas nos deparamos com a falta de registro destas informaçôes. Informaçóes sobre o número de surtos, idade do primeiro surto e data de nascimento não constavam nos prontuários de vários pacientes. Além disso, outras informaçóes estavam incompletas ou desatualizadas. Dados sobre endereço e telefone estavam incorretos e a data de nascimento estava frequentemente substituída pela idade do paciente nos registros. Contudo, essa informação (idade do paciente) não é periodicamente atualizada, dificultando o acesso a 
real idade dos pacientes. Dados como o grau de incapacidade dos pacientes também não estavam atualizados, tornando os prontuários pouco confiáveis como fonte de informação e podendo privar os profissionais de saúde de um dado potencialmente relevante. Um estudo anterior $^{20}$, que buscou avaliar a relação entre saúde mental e trabalho, já havia indicado a precariedade das anotaçóes nos prontuários e a ausência de informações relevantes nesses documentos.

Além dos problemas referentes a falta de instrumentos validados de avaliação, que ultrapassam esse relato de experiência, sendo sentidos por todos os profissionais que trabalham com avaliação psicológica ou neuropsicológica no Brasil, bem como todos os que dependem de prontuários para obter informaçóes, nossa vivência mostrou a necessidade de considerar outros aspectos ao planejar uma avaliação de pacientes com EM. Para essa população, considerar a influência do período do dia sobre a fadiga, o impacto da avaliação no estado emocional dos pacientes, sua dependência para se deslocar até o local de avaliação e a interferência familiar mostraram-se tão relevantes quanto pensar os instrumentos para a avaliação.

Alguns pacientes avaliados apresentavam dificuldades de locomoção, criando a necessidade que suas avaliaçôes ocorressem em domicílio, mas ao realizar as avaliaçóes na casa dos pacientes, tivemos que lidar com a interferência dos familiares. Alguns familiares ficavam rondando o local de avaliação, distraindo os pacientes, colocando em risco a garantia de sigilo sobre as avaliaçóes e potencialmente enviesando os resultados observados. Em mais de um caso foi necessário interromper a avaliação e pedir com insistência aos familiares que deixassem o recinto antes de retomá-la.

Outro fator de que precisa ser considerado é o horário de realização das avaliações. Como a fadiga é um sintoma frequente e se manifesta de forma mais acentuada ao fim do dia e em horários de pico de calor, várias avaliaçóes inicialmente agendadas para o período da tarde precisaram ser remanejadas para o período da manhá.

A fragilidade emocional dos pacientes também requer atenção durante o planejamento das avaliações. Em nossa avaliação, alguns pacientes apresentaram reaçôes emocionais de choro e elevada ansiedade ao perceber que não completavam as tarefas propostas de forma adequa- da, associando esse resultado a um possível agravamento da EM. A literatura ${ }^{20}$ aponta a correlação negativa entre ansiedade e a memória, indicando que a alteração emocional exacerbada vivenciada pelos pacientes durante a avaliação pode interferir no resultado de seus testes de memória. O fator emocional referente ao mau desempenho pode, ainda, ser fonte de sofrimento e interferir em outros aspectos do quadro clínico dos pacientes com EM. A literatura ${ }^{6}$ aponta uma maior vulnerabilidade para a depressão e demais doenças emocionais nos pacientes com EM, contudo, esse não tem sido um aspecto suficientemente valorizado e investigado ao planejar as baterias de avaliação ou as intervenções para essa população em nosso contexto.

\section{CONCLUSÕES}

A avaliação neuropsicológica já demonstrou seu potencial para auxiliar no refinamento do tratamento oferecido aos portadores de EM, ajudando a compreender melhor a relação entre as lesões cerebrais e os sintomas comportamentais observados nessa patologia ${ }^{5}$. Para os pacientes avaliados no presente trabalho a identificação de déficits cognitivos, de memória e motores embasaram um treinamento cognitivo que foi incorporado ao tratamento oferecido aos pacientes. Dado este potencial, faz-se necessário investir na consolidação das avaliações neuropsicológicas, realizando estudos, construindo e validando instrumentos para o contexto brasileiro, explicitando as dificuldades para realizar essas avaliações. É também necessário ampliar a discussão entre os profissionais sobre as características, carências e peculiaridades da avaliação de pessoas com síndromes crônicas ou condiçóes específicas, como a EM.

$\mathrm{Na}$ presente vivência, os instrumentos representaram a principal dificuldade enfrentada, levando a concluir que é necessário desenvolver instrumentos menos extensos para avaliar os pacientes com EM. Ao definir a bateria de avaliação os profissionais precisam, ainda, incluir a checagem do estado emocional dos pacientes. Nossa prática nos mostrou, ainda, a necessidade de manejar a interferência de aspectos característicos da EM durante as avaliaçóes, especialmente a fadiga. Recomenda-se aos profissionais que desejem avaliar pacientes com EM que marquem as avaliaçôes para o período da manhã, orga- 
nizem suas sessões de avaliação com duração máxima de uma hora por sessão e realizem um rapport para checagem de humor antes de iniciar as avaliaçóes. Sugerimos, ainda, considerar a adoção da MFSC, do teste do relógio, as provas clínicas e encontrar uma alternativa menos extensa que a BAMT-UFMG para avaliar memória. Uma opção seria utilizar um ou mais dos subtestes da Escala Weschler de Inteligência Adulta (WAIS).

Este relato refere-se apenas ao relato da avaliação neuropsicológica de um grupo de pacientes com EM de uma cidade de médio porte do interior de Minas Gerais, não podendo ser generalizado para outros contextos. Ainda assim, acreditamos que compartilhar essa experiência pode ajudar a pensar pontos relevantes para a avaliação neuropsicológica como um todo, alertando profissionais para aspectos específicos a serem considerados em seus planejamentos de ação.

\section{REFERÊNCIAS}

1.McDonald WI, Ron M. Multiple sclerosis: the disease and its manifestations. Philos TransR Soc Lond B Biol Sci 1999;354:1615-22.

http://dx.doi.org/10.1098/rstb.1999.0506

2.Callegaro D. Epidemiologia da esclerose múltipla. Trabalho apresentado no BCTRIMS First Annual Meeting, Brazilian Commitee for Treatmente and Research in Multiple Sclerosis. 2000. Salvador (BA).

3.O'Connor P. Key issues in the diagnosis and treatment of multiple sclerosis: an overview. Neurology 2002;59:1-33.

http://dx.doi.org/10.1212/WNL.59.6 suppl 3.S1

4.Paes RA, Alvarenga RMP, Vasconcelos CCF, Negreiros MA, Landeira-Fernández J. Neuropsicología de la esclerosis múltiple primaria progresiva. Rev Neurol 2009;49(7):343-8.

5.Lima EP, Haase VG, Lana-Peixoto MA. Heterogeneidade neuropsicológica na esclerose múltipla. Psicologia: Reflexão e Crítica 2008;21(1):100-9.

http://dx.doi.org/10.1590/S0102-79722008000100013

6.Ybarra MI, Teixeira Júnior AL. Transtornos depressivos e ansiosos em esclerose múltipla. Dissertaçấo de Mestrado. Universidade Federal de Minas Gerais. Belo Horizonte, MG, 2007, 59p.

7.Ferreira FO, Lima EP, Vasconcelos AG, Lana-Peixoto MA, Haase VG. Velocidade de Processamento, Sintomas Depressivos e Memória de Trabalho: Comparação entre Idosos e Portadores de Esclerose Múltipla. Psicologia: Reflexão e
Crítica 2011;24(2):367-80.

http://dx.doi.org/10.1590/S0102-79722011000200019

8.Lima EP, Rodrigues JL, Vasconcelos AG, Lana-Peixoto MA, Haase VG. Heterogeneidade dos déficits cognitivo e motor na esclerose múltipla: um estudo com a MSFC. Psico. 2008;39(3):371-81.

9.Mendes MF, Tilbery CP, Balsimelli S, Moreira MA, Cruz AMB. Teste de destreza manual da caixa e blocos em indivíduos normais e em pacientes com esclerose múltipla. Arq Neuropsiquiatr 2001;59(4):889-94.

http://dx.doi.org/10.1590/S0004-282X2001000600010

10.Oliveira EML, Annes M, Oliveira ASB, Gabbai AA. Estudo clínico de 50 pacientes acompanhados no ambulatório de neurologia UNIFESP-EPM. Arq Neuropsiquiatr 1999;5(1):63-7.

11.Lei Ordinária no 4119 de 27 de agosto de 1962. Dispóe Sobre os Cursos de Formação em Psicologia e Regulamenta a Profissão de Psicólogo. Governo Federal do Brasil (citado em: 03/2012; atualizado em: 02/2013). Disponível em: http://br.vlex.com/vid/cursos-psicologia-regulamenta-psicologo-34144071.

12.Lei Federal no 5.766 de 20 de dezembro de 1971. Cria o Conselho Federal e os Conselhos Regionais de Psicologia e dá outras providências. Governo Federal do Brasil (citado em: 03/2012; atualizado em: 02/2013). Disponível em: http://www.jusbrasil.com.br/legislacao/128508/lei-5766-71.

13.Resoluçấo CFP 002/2003. Define e regulamenta o uso, a elaboração e a comercialização de testes psicológicos e revoga a Resolução CFP nº 025/2001. Conselho Federal de Psicologia (citado em: 04/2012; atualizado em: 02/2013). Disponível em: http://www.pol.org.br/pol/export/sites/default/pol/legislacao/ legislacaoDocumentos/resolucao2003_02.pdf.

14.Código de Ética Profissional dos profissionais da Psicologia. Conselho Federal de Psicologia (citado em: 04/2012; atualizado em: 02/2013). Disponível em: http://www.pol.org.br/pol/export/sites/default/pol/legislacao/legislacaoDocumentos/codigo_etica.pdf.

15. Wood GMO, Carvalho MRS, Rothe-Neves R, Haase VG. Validação da Bateria de Avaliação da Memória de Trabalho (BAMT-UFMG). Psicologia: Reflexão e Crítica 2001;14(2):325-41.

http://dx.doi.org/10.1590/S0102-79722001000200008

16. Haase VG, Lima EP, Lacerda SS, Lana-Peixoto MA. Desenvolvimento da versão brasileira da multiple sclerosis funcional composite measure (MSFC-BCTRIMS). Arq Neuropsiquiatr 2004;62(2-A):363-70. http://dx.doi.org/10.1590/S0004-282X2004000200033

17.Sunderland T, Hill JL, Mellow AM. Clock drawing in Alzheimer's disease. A novel measure of dementia severity. J Am Geriatr Soc 1989;37:725-9.

18.Atalaia-Silva KC, Lourenço RA. Tradução, adaptação e validação de construto do Teste do Relógio aplicado entre idosos no Brasil. Rev Saúde Pública 2008;42(5):930-7.

http://dx.doi.org/10.1590/S0034-89102008000500020

19.Paulo DLV, Yassuda MS. Queixas de memória de idosos e sua relação com escolaridade, desempenho cognitivo e sintomas de depressão e ansiedade. Rev Psiq Clín 2010;37(1):23-6.

http://dx.doi.org/10.1590/S0101-60832010000100005

20.Lima MEA. A relação entre distúrbio mental e trabalho - evidência epidemiológica recentes. In: Wanderley Codo (Org.). O trabalho enlouquece? Um encontro entre a clínica e o trabalho. 1ed. Petrópolis: Vozes, 2004, p.139-60. 